\title{
The Case and Epidemiological Analysis of Low Vision in the Aged People
}

\author{
Xiao Zhang ${ }^{1}$, Yan Liu ${ }^{2}$ \\ ${ }^{1}$ North China University of Science and Technology, 063000 \\ ${ }^{2}$ Affiliated Hospital of North China University of Science and Technology, 063000
}

Keywords: aged people; low vision; causes; Epidemiology

\begin{abstract}
Objective: To investigate the causes of low vision in aged patients and to explore the clinical features of these patients, in order to provide evidences for protecting eyesight and improving quality of life in elderly people. Methods: A total of 310 patients $\geq 60$ years of age with low vision were collected from our hospital between January, 2016 to December, 2017 to fill the basic questionnaires, and the distance, near vision, slit lamp, intraocular pressure, fundus and retinoscopy of aged people were checked. Results: The main causes of vision loss in aged people in 320 patients were refractive errors, accounting for $61.93 \%$; cataracts second, accounting for 22.10\%; glaucoma accounting for 5.64\%; retinal lesions accounting for $4.19 \%$; maculopathy accounting for $4.19 \%$; other diseases accounted for $1.93 \%$. The degree of vision loss was serious with age increased, the Spearson correlation coefficient was 0.203 ( $\mathrm{P}<0.001$ ). Patients with diabetes $\geq 5$ years had worse visual acuity than those $<5$ years with statistically significant differences (Chi square $=10.496, \mathrm{P}=0.005$ ). Conclusion: The causes of vision loss in aged people are complex. To protect the eyesight of the aged people, targeted measures should be taken.
\end{abstract}

\section{Introduction}

China has a large population base and is one of the most populous countries in the elderly. At present, the proportion of the elderly population over 60 years of age in China is increasing gradually. and it is estimated that it will reach about $16 \%$ of the population by $2020^{[1]}$. With the increase of the proportion of the elderly, the health of the elderly is gradually getting attention. The loss of vision in the elderly affects the daily life of the elderly, like other diseases, which can reduce the quality of life of the elderly, as well as the social and economic burden. Research at home and abroad have shown that vision loss in the elderly is age-related, and older people have lower vision ${ }^{[2,3]}$. As a public health problem, the vision loss of old people deserves the attention of the society.

With the socio-economic and health development, concerns about the problem of vision loss and quality of life of the elderly are put on the agenda. Through investigation, we can understand the incidence and causes of vision loss in the elderly, which is helpful for early prevention, timely treatment, and rehabilitation training as early as possible. Common causes of vision loss in the elderly are hypermetropia and myopia, glaucoma, senile cataracts, retinopathy, etc ${ }^{[4]}$. In order to grasp the cause of vision loss in the elderly, protect the vision and enhance life quality of old people. We conducted a questionnaire and systematic eye examinations of $\geq 60$-year-olds who visited our hospital from January 2016 to December 2017 to understand and grasp the causes of the vision loss in the elderly population. It will help ophthalmologists to correct the cause of the disease and improve their visual acuity and quality of life as soon as possible.

\section{Survey Object and Method}

\subsection{Survey object}

We collected 320 patients aged over 60 who had vision loss from January 2016 to December 2017 in our hospital, and using the status quo survey method to carry out the questionnaire survey and visual acuity study. The subjects included in the study were older than 60 years old, and they 
were able to cooperate with the eye examination and take the voluntary principle, The exclusion criteria are unclear awareness, normal expression, hearing impairment, or inability to cooperate with the inspectors.

\subsection{Method}

We conducted a survey of selected subjects using a self-designed questionnaire that included information on name, gender, age, occupation, current medical history, and past medical history. Routine ophthalmology examinations were performed, including far and near nude eyes, slit lights, intraocular pressure, and fundus examination. To monocular uncorrected visual acuity $\geq 5.0$ for normal vision, $<5.0$ for visual acuity. The visual acuity was divided into three groups, which were 4.9 4.6, 4.5 4.1 and <4.0. When there is inconsistency of binocular vision to lower visual acuity prevail, and hypopsia is monocular visual acuity lower than the previous determination.

Uncorrected visual acuity test method: An international standard visual acuity chart was used to examine the Binocular distant vision of the subjects. The ophthalmologist used the international standard visual acuity chart to carry out the examination in the distance of $5 \mathrm{~m}$, and all the subjects were recorded as the result of the visual acuity of the least 1 line of the one letter. If the subject does not wear corrective glasses with far vision, check uncorrected visual acuity. If the subjects wear far corrected glasses, their visual acuity is checked after the examiner wears glasses.

Outer eye examination: Check eye movements, eye position, eyelids, eyelashes, lacrimal devices, eyelid conjunctiva and cornea in natural light or flashlight light and observe whether corneal ulcers, leukoplakia and so on. Lens examination was performed under slit lamp. The fundus examination was performed directly under the small pupil to observe whether there were bleeding, exudation, and hiatus.

\subsection{Statistical Analysis}

Epidata3.0 data entry, using SPSS13.0 for statistical analysis, qualitative data using $\chi 2$ test, all $\mathrm{P}$ values represent bilateral probability, the test level a is 0.05

\section{Result}

\subsection{Basic situation}

A total of 310 patients $\geq 60$ years of age with vision loss from January 2016 to December 2017 in our hospital were collected. The patients (aged 60-88 years), mean age 71 years, were enrolled in this study. 150 cases of male total of 300 eyes, 160 women a total of 320 eyes.

\subsection{Vision loss and ophthalmopathy distribution}

As can be seen from Table 1, the main causes of vision loss in the elderly are refractive errors, accounting for $61.93 \%$; cataracts second, accounting for $22.10 \%$; glaucoma $5.64 \%$, retinopathy 4.19\%; macular degeneration 4.19\%; other diseases Species accounted for 1.93\%. See Table 1.

Table 1 Elderly people with vision loss and the distribution of ophthalmopathy

\begin{tabular}{lll}
\hline Diseases & The number of diseased eyes & Composition ratio(\%) \\
\hline Refractive error & 384 & 61.93 \\
cataract & 137 & 22.10 \\
glaucoma & 35 & 5.64 \\
Retinopathy & 26 & 4.19 \\
Macular lesion & 26 & 4.19 \\
Other diseases Species & 12 & 1.93 \\
total & 620 & 100 \\
\hline
\end{tabular}

\subsection{Relationship between vision loss and refractive error}

In ametropia, hyperopia and astigmatism accounted for the largest proportion (58.59\%), astigmatism accounted for 7.81\%; myopia and astigmatism accounted for 33.58\%; corrected visual 
acuity 5.0 to 4.9 accounted for $32.81 \%$, 4.8 to accounted for $39.58 \%$, $4.3 \sim 4.1$ accounted for $19.53 \%,<4.0$ accounted for $8.07 \%$.

Table 2 Elderly people with vision loss and refractive error (\%)

\begin{tabular}{cccccc}
\hline Refractive & The number of & \multicolumn{4}{c}{ Corrected vision } \\
\cline { 3 - 6 } error & ophthalmopathy & $5.0 \sim 4.9$ & $4.8 \sim 4.4$ & $4.3 \sim 4.1$ & $<4.0$ \\
\hline myopia & $76(19.79)$ & $22(28.95)$ & $37(48.68)$ & $12(15.79)$ & $5(6.58)$ \\
$\begin{array}{c}\text { Myopia with } \\
\text { astigmatism }\end{array}$ & $53(13.80)$ & $11(20.75)$ & $26(49.05)$ & $13(24.53)$ & $3(5.66)$ \\
$\begin{array}{c}\text { Hyperopia } \\
\begin{array}{c}\text { Hyperopia } \\
\text { with }\end{array}\end{array}$ & $119(30.99)$ & $56(47.06)$ & $37(31.09)$ & $22(18.49)$ & $4(3.36)$ \\
$\begin{array}{c}\text { astigmatism } \\
\text { mixed }\end{array}$ & $106(27.60)$ & $32(30.19)$ & $41(38.68)$ & $19(17.92)$ & $14(13.21)$ \\
$\begin{array}{c}\text { astigmatism } \\
\text { total }\end{array}$ & $30(7.81)$ & $5(16.67)$ & $11(36.67)$ & $9(30.00)$ & $5(16.67)$ \\
\hline
\end{tabular}

\subsection{Relationship between vision loss and age}

As can be drawn from Table 3, the aged people with vision loss accounted for $38.71 \%$ of all patients aged $60-64$ years, $32.23 \%$ of those aged $65-69$ years and $27.74 \%$ of those aged $70-70$ years. The degree of vision loss was positively correlated with age. The worse the vision loss with age, the Spearson correlation coefficient was $0.203(\mathrm{P}<0.001)$.

Table 3 Elderly people with vision loss and age (\%)

\begin{tabular}{ccccc}
\hline age & Number of & \multicolumn{3}{c}{ vision } \\
\cline { 3 - 5 } & cases & $4.9 \sim 4.6$ & $4.5 \sim 4.1$ & $<4.0$ \\
\hline $60 \sim 64$ & $121(38.71)$ & $89(73.55)$ & $20(16.53)$ & $12(9.92)$ \\
$65 \sim 69$ & $103(32.23)$ & $67(65.05)$ & $20(19.42)$ & $16(15.53)$ \\
$\geq 70$ & $86(27.74)$ & $41(47.67)$ & $22(25.58)$ & $20(23.26)$ \\
\hline total & $310(100)$ & $225(72.58)$ & $57(18.39)$ & $28(9.03)$ \\
\hline
\end{tabular}

\subsection{Relationship between vision loss and hypertension and diabetes in the elderly}

As can be seen from Table 4, 137 of the 310 respondents (44.19\%) were hypertensive patients and 65 (20.97\%) were diabetic patients; Hypertensive patients in the course of $<5$ years and $\geq 5$ years, there was no significant difference between the vision loss (Chi square $=0.389, \mathrm{P}=0.841$ ); Diabetes course more than 5 years in patients with serious vision loss less than 5 of the patients, the difference was statistically significant difference $(=10.496$, chi square $\mathrm{P}=0.005)$.

Table 4 Elderly people with vision loss and hypertension, diabetes (\%)

\begin{tabular}{|c|c|c|c|c|c|}
\hline \multirow{2}{*}{$\begin{array}{l}\text { history of past } \\
\text { illness }\end{array}$} & \multirow{2}{*}{$\begin{array}{c}\text { course } \\
\text { of } \\
\text { disease }\end{array}$} & \multirow{2}{*}{$\begin{array}{c}\text { Number of } \\
\text { cases }\end{array}$} & \multicolumn{3}{|c|}{ vision } \\
\hline & & & $4.9 \sim 4.6$ & $4.5 \sim 4.1$ & $<4.0$ \\
\hline \multirow[t]{2}{*}{ hypertension } & $<5$ years & 81(59.12) & 33(37.08) & $25(30.86)$ & $23(28.39)$ \\
\hline & $\geq 5$ years & $56(40.88)$ & 20(35.71) & $18(32.14)$ & $18(32.14)$ \\
\hline Total & & $137(100)$ & 53(38.68) & 43(31.37) & 41(29.93) \\
\hline \multirow{2}{*}{$\begin{array}{l}\text { diabetes } \\
\text { mellitus }\end{array}$} & $<5$ years & $38(58.46)$ & 18(47.37) & $13(34.21)$ & $7(18.42)$ \\
\hline & $\geq 5$ years & $27(41.54)$ & $5(18.52)$ & 7(25.93) & $15(55.56)$ \\
\hline Total & & $65(100)$ & 23(35.38) & $20(30.77)$ & 22(33.85) \\
\hline
\end{tabular}

Elderly organs and organs of the body aging with age, of which the elderly eye changes in addition to changes in the structure of the eye may also occur functional disorders such as visual acuity, seriously affecting the daily life of older people Life, reduce their quality of life ${ }^{[5]}$. The common reason of senile vision decline is physiological factors, With the gradual decline of physiological function with age, the function of ocular organs will also appear aging with the aging 
of the body. two is the pathological reasons, such as cataract, glaucoma, retinopathy and other diseases ${ }^{[6]}$.

The survey results show that: the main reason for vision loss in the elderly is refractive error, accounting for $61.93 \%$; followed by cataract accounted for $22.10 \%$, glaucoma $5.64 \%$, retinopathy 5.64\%; macular degeneration accounted for 4.19\%; other diseases Accounting for $1.93 \%$. Elderly mild hyperopia can appear compensatory adjustment, visual acuity can be normal ${ }^{[7]}$.If the body can not be compensatory adjustment will appear absolute hyperopia, eventually varying degrees of vision loss. The visual fatigue symptoms can disappear or significantly reduce after the visual adjustment of the convex lens ${ }^{[8,9]}$.

It is reported in the literature that age is the influential factor of ametropia in the elderly ${ }^{[10]}$. With the increase of age, the regulation function of ciliary muscle decreased, which turned recessive hyperopia into dominant hyperopia, and showed signs of visual acuity decline. secondly, with the increase of age, the lens sclerosis gradually, the elasticity decreases, and the thickness of lens increases, which results in the change of refractive state. Patients with refractive errors can improve their quality of life by taking a combination of optometry and wearing appropriate glasses to achieve normal or significant improvement in vision ${ }^{[6,11]}$. Another reason for the decline in vision loss with increasing age is the increased incidence of cataract with increasing age. The best treatment for such patients is surgery, but it increases the financial burden on the family and the community. Therefore, the method of preventing senile cataract is to pay attention to prevention, to pay attention to the examination of visual acuity in the elderly, to avoid the missed diagnosis and misdiagnosis of ametropia. An important step in the prevention and treatment of vision loss in the elderly is to wear glasses for vision correction based on the results of the examination. In addition, with the increase of age, the number of patients with diabetes and hypertension increased correspondingly, and the corresponding eye complications also increased, which became a major risk factor for vision loss in the elderly. Such patients should be actively treated for related diseases, and patients with impaired vision should improve their vision by optometry and wearing appropriate glasses.

The study also found that vision loss in the elderly is positively correlated with age, the older the age, the more serious the vision decline.; about $21.0 \%$ of patients with vision loss suffering from diabetes, the longer the course of diabetes, the more obvious the vision loss. Therefore, patients with diabetes should reduce the incidence of visual impairment or reduce the degree of visual impairment by controlling blood glucose. When finding visually impaired elderly patients with refractive errors, medical institutions should first consider the cause of refractive errors, and then make a correct diagnosis based on the patient's medical history to reduce misdiagnosis.

\section{Discussion and Conclusion}

In summary, the causes of vision loss in the elderly are complicated. In this study, we found that the main cause of vision loss in the elderly is refractive errors. In addition, there are cataract and glaucoma. Therefore, health education should be strengthened for the elderly on eye health knowledge, and improve the overall vision of the elderly by treating the causes of vision loss.In addition, we should make census work for the elderly such as glaucoma and cataracts and treat them actively. And through a variety of measures to reduce the incidence of visual loss of the elderly, through treatment to improve visual acuity and then improve the quality of life.

\section{References}

[1] Zhonglin Yu, Shanbi Zhou.40 cases of elderly patients with vision loss causes [J]. Journal of Chongqing Medical University, 2000,25 (1): 90-91

[2] Lili Wang. Hequ City, Guangxi Jinchengjiang elderly vision survey [J]. Huaxia Medicine, 5 (22): 939-942

[3] Yuanyuan Fan, Guolier Qiduer, Bin Wei. A survey of blindness and low vision in 50 years old 
and above aged apartment in Urumqi[J]. Ophthalmology, 2011,20 (2): 133-136

[4] Li Huang. Analysis of causes of vision loss in middle-aged and old people [J]. Chinese Geriatric Health Medicine, 2009,7 (3): 43-44

[5] Luo Bu-ren. Etiological analysis of 220 elderly patients with vision loss in Tibet [J]. China Otorhinolaryngology, 2007,7 (6): 391

[6] Lirong Lu, Zhiwei XU, Jintao Chen. Analysis of family support status and influencing factors in elderly patients with low vision [J]. Zhejiang Preventive Medicine, 2011,23 (12): 19-21

[7] Qun Xia, Xiaoping Guo, Wei Zhang. Abnormal vision and age-related eye diseases[J]. Chinese Journal of Gerontology, 2006,26 (12): 1729-1730

[8] Xu L, Cui T, Yang $\mathrm{H}$, et al. Prevalence of visual impairment among adults in China: The Beijing Eye study[J]. Am J Ophthalmol,2006,141(3):591-593

[9] Wuhe Chen. Opportunities and Challenges for Low Vision Rehabilitation in China[J]. Chinese Journal of Optometry and Visual Science, 2010,1 (3): 167

[10] Gu Liqimu· Aimaier, Songjie Liao, Xiaoli Yao, Qi Wu, Zhongyuan Lai, Yang Feng. Misdiagnosis analysis of neurosyphilis with vision loss as the only manifestation[J]. Clinical misdiagnosis and mistreatment, 2017,265 (09): 1-3.

[11] Aihua Chen. Risk factors for vision loss in young patients with phacoemulsification[J]. Modern Diagnosis and Treatment, 2017,231 (03): 563-565. 